\title{
Degradation of perovskites and Dexter-Varley paradox
}

\author{
B. L. Oksengendler ${ }^{1,2}$, S. E. Maksimov², M. B. Marasulov ${ }^{1}$ \\ ${ }^{1}$ Scientific Center of Chemistry and Physics of Polymers at National University \\ of Uzbekistan, Tashkent, Uzbekistan \\ ${ }^{2}$ Institute of Ion-Plasma and Laser Technologies, Uzbek Academy of Sciences, \\ Tashkent, Uzbekistan \\ oksengendlerbl@yandex.ru,maksimov_s@yahoo.com
}

\section{PACS 75.50.Tt, 81.16.Hc, 87.85.Rs $\quad$ DOI 10.17586/2220-8054-2015-6-6-825-832}

This work presents a model for the degradation mechanism of organic-inorganic hybrid photovoltaic solar cells based on perovskites. The cross section for formation of Frenkel pairs in the sublattice of iodine is obtained. The channels for its annealing are found. Special attention is paid to the polaron states. It is shown that the stationary number of defects non-monotonically depends on the intensity of solar radiation. This allows one to analyze the properties of the radiation resistance of a device to ionizing radiation.

Keywords: perovskites, degradation, ionizing irradiation, Varley mechanism, polaronic states, organic solar cells.

Received: 1 November 2015

\section{Introduction}

Perovskite based solar-cells have attracted much attention due to their ever-increasing conversion efficiency. In the most general case, typical perovskite materials have a structure which is $\mathrm{CH}_{3} \mathrm{NH}_{3} \mathrm{PbJ}_{3}$ or close variants [1]. The start of the "perovskite era" (see [2-5]) occurred in 2009; then, over 2012-2013 the conversion rate of solar energy into electrical energy increased from $10 \%$ to $15 \%$. To date, the characteristic physical and mechanical structure of a perovskite solar cell (Figs. 1a, 1b, 1c, 1d) has been formed, which is characterized by the corresponding electronic band structure of the device (Fig. 1b). Along with excellent features of perovskite cells (inexpensive and relatively simple low-temperature technology and an understandable physical chemistry of the components), some difficulties have been identified, whose solution could lead, according to some experts [2], to a "new era of photovoltaics": these are the very small sizes of the solar cells $\left(<1 \mathrm{~cm}^{2}\right)$, environmental issues (dominant concentration of $\mathrm{Pb}$ ), and issues of the stability of solar cells. The latter aspect, which consists of the interaction with the humidity in the environment (more precisely, with hydroxyl ions, $\mathrm{OH}^{-}$), redox reactions involving oxygen, and the radiation stability of the working solar element, is particularly important and actually has not been studied (except for one experimental work [6]). The present work aims to examine this problem, namely, a theoretical analysis of the impacts of ultraviolet radiation on perovskite solar cells.

\section{Generation of Defects}

It follows from the most common considerations of radiation physics for solids (see [7, 8]), that the most sensitive element to ultraviolet radiation is perovskite, which belongs to a class of semiconductors. The band structure of this element is shown in the central part of Fig.1. The entire set of radiative processes affecting the electronic spectrum is reduced to the generation of radiation defects, their radiation-enhanced diffusion, quasi-chemical reactions, 
(a)

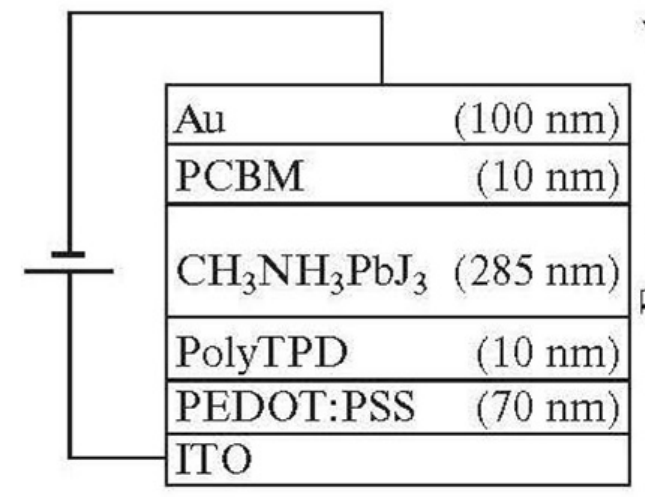

(c)

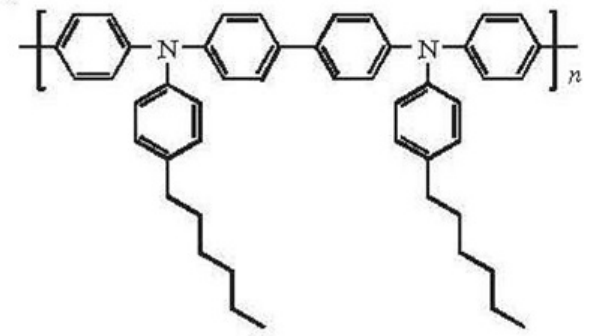

(b)

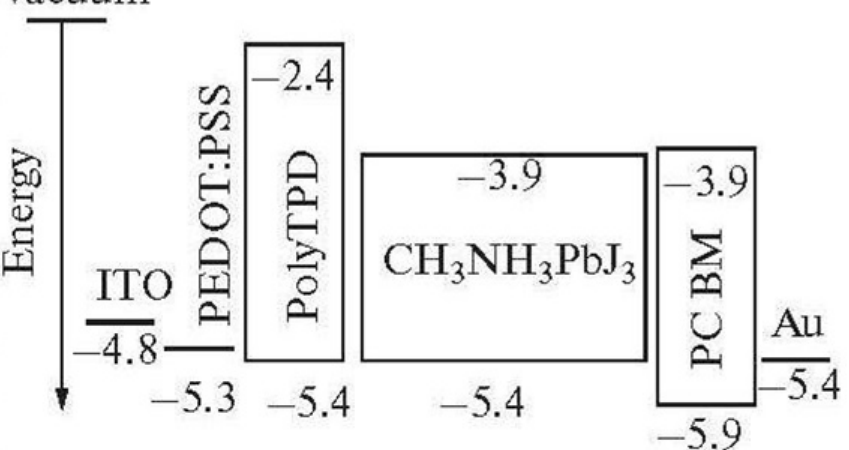

(d)

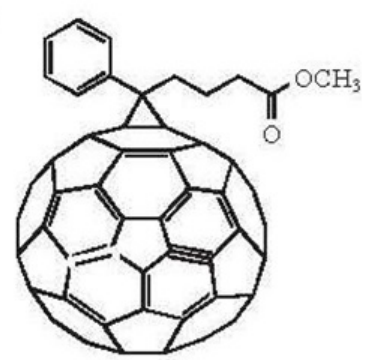

FIG. 1. The scheme of a solar cell based on perovskite: (a) the geometric structure of layers; (b) the electronic structure of layers; (c) the chemical structure of polerelomin (polyTPD); (d) the chemical structure of PCBM (see $[1-3])$

and the charge transfer (especially at the interface) of emerging local level modifications. Thus, the basic radiation phenomena producing the electronic spectrum of perovskite are reduced to the emergence of defects and their annealing.

\section{The case of UV-irradiation}

Varley-type mechanisms are the most important pathway for defect formation in perovskite-type materials $[9,10]$, since the elastic displacements are prevented due to the large difference between the masses of $\mathrm{I}$ and $\mathrm{Pb}$ atoms with the masses of bombarding particles. The scheme of the Varley mechanism applied to perovskite, $\mathrm{CH}_{3} \mathrm{NH}_{3} \mathrm{PbI}_{3}$, is as follows. In the first stage ionization of the subvalent shell of the I atom, ultraviolet radiation occurs (ses Fig. 2). In the second stage, the Auger process, which consists of a hole in the $\mathrm{J}$ subvalent shell and two electrons of the valence band constructed of 5 p-functions of the $I^{-}$ion (see [11]) occurs. As a result of this process, a singly charged ion is formed at the position of iodine (the probability of such a process is defined as $\alpha_{\text {Auger }}$ ) (Fig. 3).

In the third stage, the resulting positive $\mathrm{I}^{+}$ion, which is surrounded by positive $\mathrm{Pb}^{n+}$ ions that constitute perovskite, is on the precipice of the positive potential of the lattice of the inversion effect of the Madelung potential instead of being in the potential well; this state is unstable and is attributed, according to the classification of radiation physics, to dynamic instability [7, 8] (Fig.4).

The fourth stage implies dynamically unstable configurations of $I_{S}^{+}$relax due to two competing processes: either by the offset of $I_{S}$ to the nearest interstitial configuration (that 


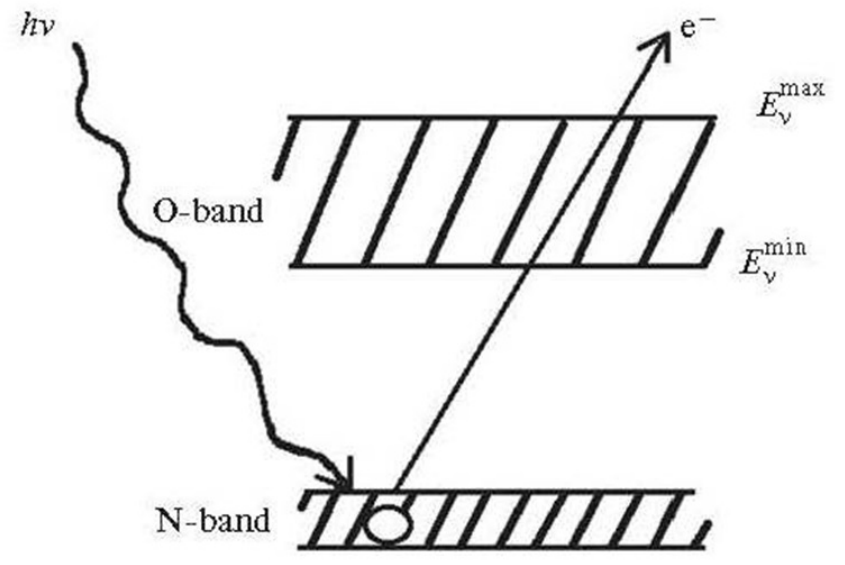

FIG. 2. The formation of a hole in the N-band of perovskite during the absorption of a photon

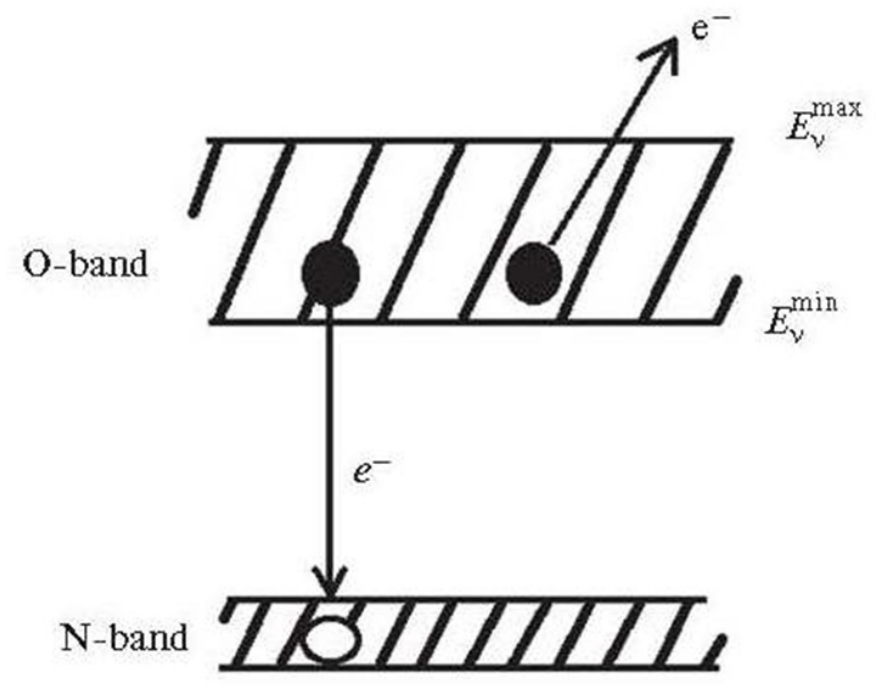

FIG. 3. The formation of two holes in the O-band of perovskite as a result of the Auger-process

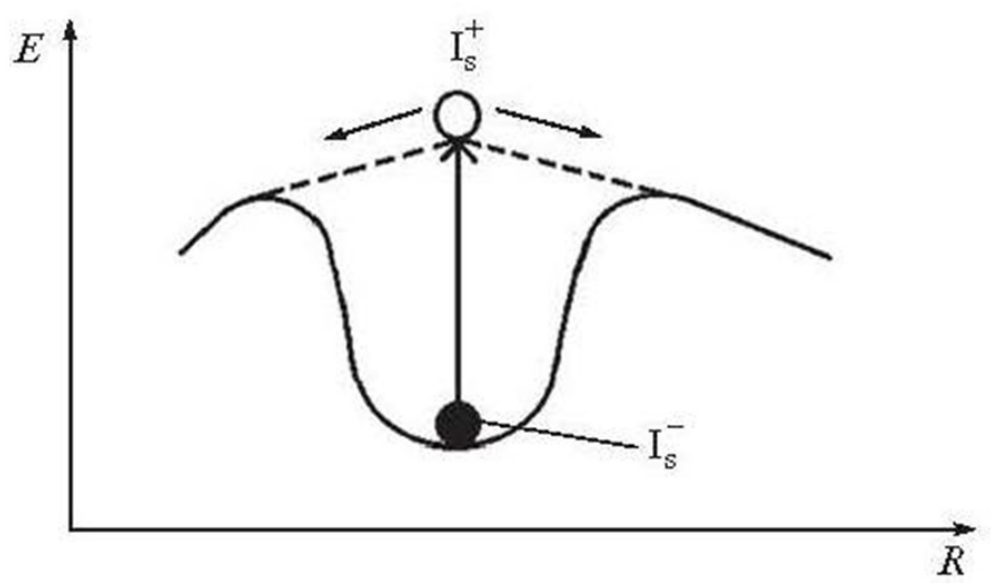

FIG. 4. The formation of dynamic Coulomb instability in the I-sublattice of perovskite 
is, a defect via the reaction $I_{S}^{+} \rightarrow V_{I}+J_{i}^{+}$is formed, where $V_{I}$ is the iodine vacancy, $I_{s}$ and $I_{i}$ are nodular and interstitial positions), or iodine ion neutralization $I_{S}^{+}+e \rightarrow I_{S}^{0}$ thus, a Frenkel pair is not formed. The last step has probabilistic nature and the likelihood of the offset of the atom into the interstice is determined by the expression (Fig. 5):

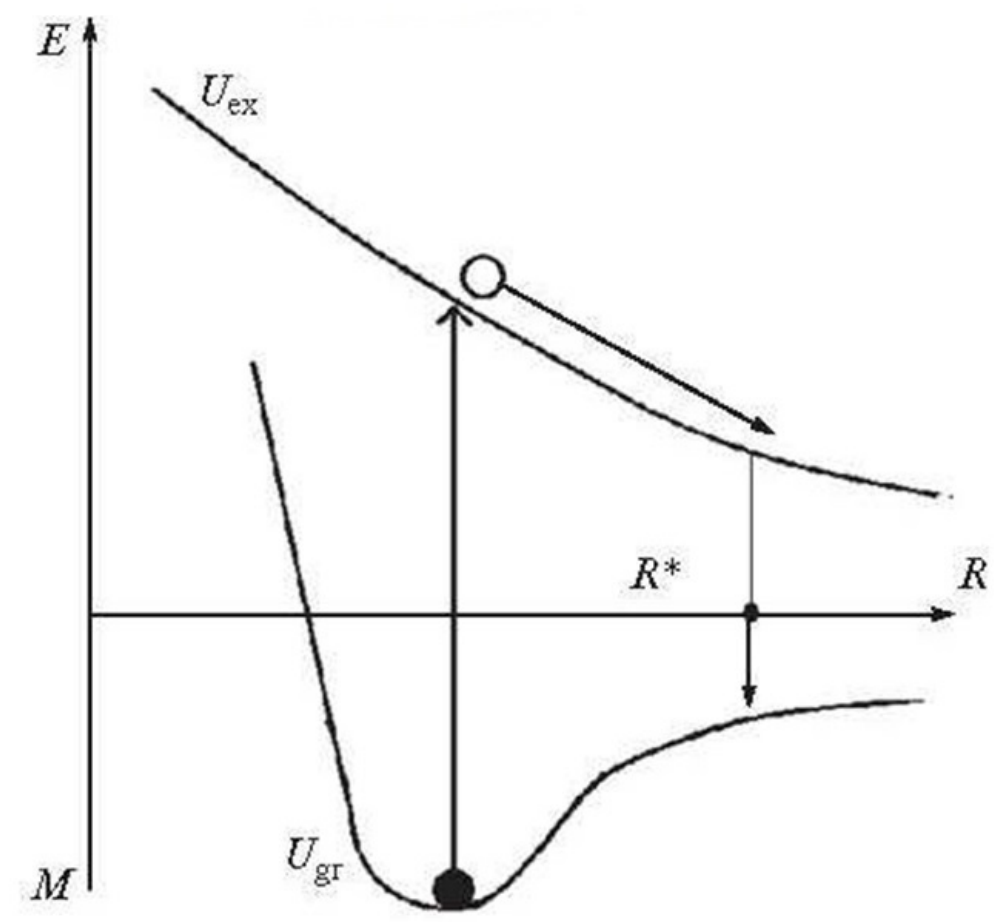

FIG. 5. The decay of an unstable local domain in the I-sublattice of perovskite

$$
\eta=\exp \left(-\tau_{+} / \tau_{e}\right)
$$

where $\tau_{+} \approx 5 \times 10^{-14} \mathrm{~s}$ is the time of critical offset for the ion and $1 / \tau_{e}$ is the probability of neutralizing the positive charge that fell into the valence band of perovskite as a result of the Auger process. Calculation of $1 / \tau_{e}$ must consider two channels of neutralization: the expansion of two holes, which are localized on one node of iodine as a result of the Auger transition, over the valence band $\left(1 / \tau_{e 1}\right)$ and capture by the excess charge on $I_{S}^{+}$of the conduction band electrons that occur there due to the absorption of photons in the perovskite layer $\left(1 / \tau_{e 2}\right)$. Calculations show [12-15] that $1 / \tau_{e 1}=\mu e / a^{3}$, where $\mu$ is the mobility of holes in the valence band that is formed by regular $I^{-}$iodine ions, e is the charge of the hole, and $a$ is the distance between ions in the iodine sublattice. On the other hand, the second channel, where free electrons neutralize the conduction band, is a cavitation process [12] and the likelihood of the process is given by $1 / \tau_{e 2}=A n^{5 / 12}=\tilde{A} n^{1 / 2}$, that is, it is symbatic to light pumping (here $A$ and $\tilde{A}$ are the parameters of the material and the values of the positive charge introduced into the band by the Auger transition).

Finally, the cross section for the generation of the Frenkel pairs during the exposure to ultraviolet radiation is given by:

$$
\sigma_{d}=\sigma_{i} \alpha_{\text {Auger }} \exp \left(-\tau_{+} / \tau_{e}\right) .
$$

Assessment of this value at $\sigma_{i} \approx 10^{-17} \mathrm{~cm}^{2}, \alpha_{\text {Auger }} \approx 0.5, \tau_{e} \approx 5 \times 10^{-15}$ s gives $\sigma_{d} \approx$ $5 \times 10^{-22} \mathrm{~cm}^{2}$, that is, it is greater by four orders of magnitude than the similar value for the 
Si crystal under X-ray irradiation $[10,14]$. In addition to this unexpected result, let us note an important fact: $\sigma_{d} \approx \exp \left(-\right.$ const $\left.^{*} n^{1 / 2}\right)$, indicating an increase in the radiation resistance of the solar cell based on perovskite under intensive light fluxes (Fig. 6).

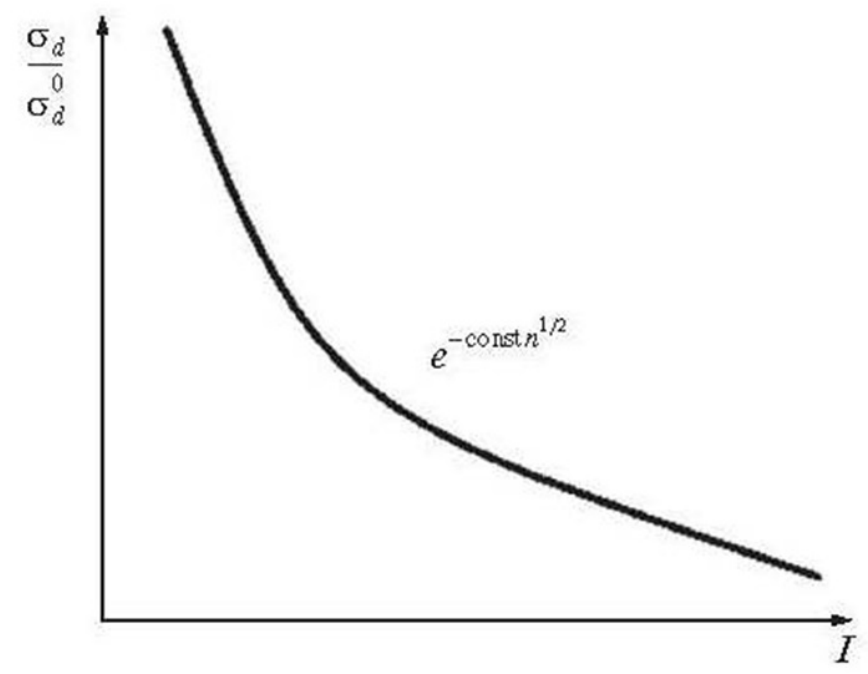

Fig. 6. The reduction of the normalized cross section of Frenkel defect formation in the I-sublattice on radiation intensity

\section{The case of the light irradiation}

The sequence of steps for defect formation is modified under the action of light (solar) irradiation. Indeed, the hole is formed at the first step directly in the valence band of perovskite (the cross-section of process is $\sigma_{i}$ ), so the Auger process with the involvement of the hole in subvalent shell falls off. Formally, this circumstance is reflected in the formula (2) as $\alpha_{\text {Auger }}=1$, and $\sigma_{i}$ corresponds to the photon disruption of the electron from the $J_{S}^{-}$ ion. In addition, the relaxation channel for the single-site hole state into the delocalized state is a type of autoionization. So again, $\eta=\exp \left(-\tau_{+} / \tau_{e}\right)$, however, for the $1 / \tau_{e}$, another estimation $1 / \tau_{e}=\Delta E_{v / \hbar}+\tilde{A} n^{1 / 2}$ will be valid, where $\Delta E_{v}$ is the width of the valence band. Finally, we obtain: $\sigma_{d}=\sigma_{i} \exp \left(-\tau_{+} / \tau_{e}\right)$.

\section{The possible role of the polaron effect in the degradation enhancement}

Dependency of $1 / \tau_{e}$ upon $\Delta E_{v}$ poses a very interesting question about the role of a strong polarization effect due to the presence of an organic dipole $\left(\mathrm{CH}_{3}-\mathrm{NH}_{3}^{+}\right)$in the perovskite. According to Prof. A.Zakhidov (Texas), electrons and holes in the perovskite turn into polarons. Without specifying what type of polarons are formed in the perovskite, it can be assumed that the effective mass of carriers will increase in this case (eg., by $m_{\text {eff }}=m_{0}(1+\tilde{\alpha} / 6)$, where $\tilde{\alpha}$ is the parameter for the electron-ion interaction [15]). The latter condition reduces $1 / \tau_{e}$ rapidly, which leads to an increase in the defect formation crosssection as $\sigma_{d} \sim \exp (-$ const $/ \tilde{\alpha})$. Moreover, it is interesting to note that the formation of biexciton states is also possible at a certain ratio of the material parameters (including the single-site ones in the so-called U-negative states [17]). Then, this can be expected to 
increase the degradation under UV - irradiation action, since $1 / \tau_{e}$ decreases sharply in this case.

\section{Varley mechanism at the interfaces}

The above-described mechanism for ionization defect formation in perovskites has a number of features at the interfaces. Primarily, it is due to the fact that $\Delta E_{v}$ is connected on the surface with the Tamm states band and is much smaller than $\Delta E_{v}$ in the bulk $E_{v}^{S}<<\Delta E_{v}^{b}[18]$. This leads to the following consequences: the scheme of the ordinary (non-radiation) degradation is effective, in which the surface is very important factor for photochemical reaction [19]. At the same time, however, it is necessary to take into account the reduction in these reactions' efficiencies according to the formula $\eta=\exp \left(-\tau_{+} / \tau_{e}\right)$. In the problem of the ionization-stimulated desorption, which absolutely has not been studied yet for perovskites, the existence of highly polarizing $\mathrm{CH}_{3}-\mathrm{NH}_{3}^{+}$components makes very interesting the appearance of bipolar hole U-states, occurring after the primary ionization of the subvalent shell of surface $J^{-}$ion; here, we can expect modifications of Knotek-Feibelman process (KF) [20] (for instance, the deepening of KF-state).

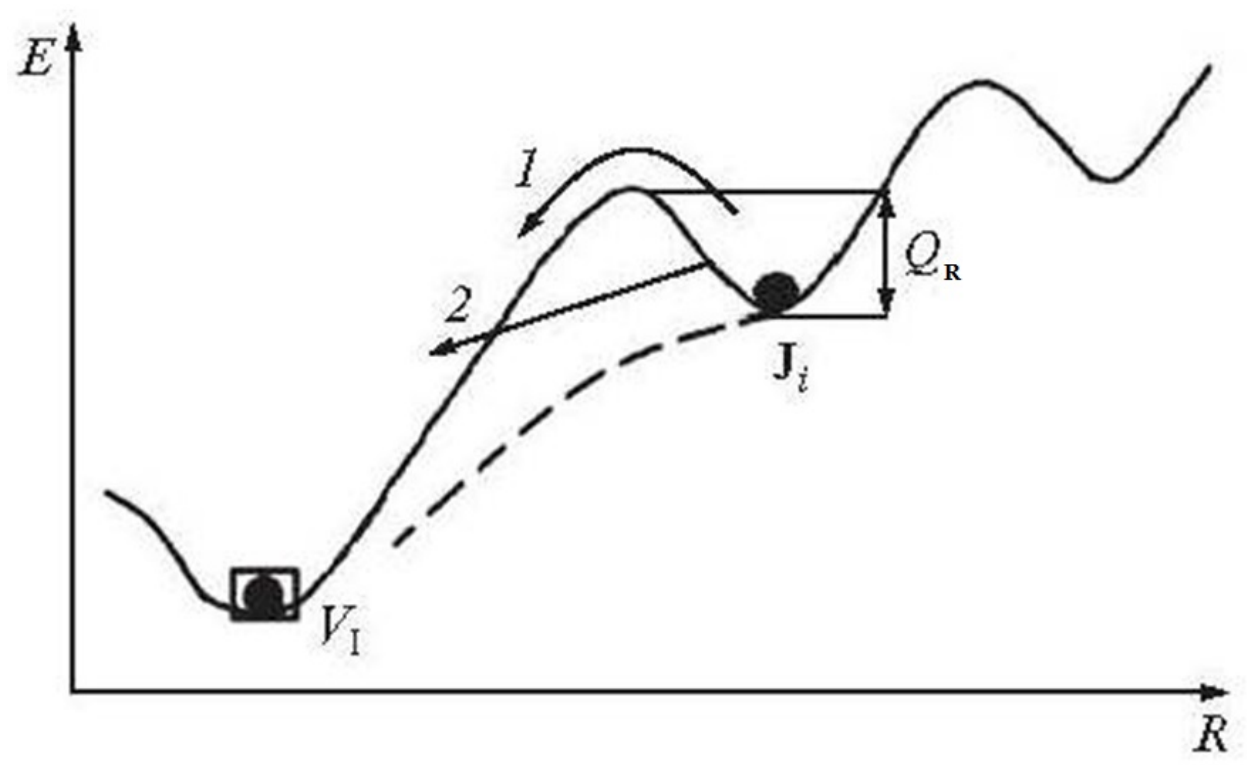

FIG. 7. The channels of the annealing of close Frenkel pair in the I-sublattice:

(1) thermally activated channel; (2) recombination-stimulated channel

\section{Annealing of defects}

Under ionizing radiation, only the bound Frenkel pairs can be formed in the iodine sublattice (Fig.7). This results in a distinctiveness for the defect annealing process: a direct (single) jump of the iodine atom shifted into the interstice in the opened vacancy via the following reaction $I_{i}^{-}+V_{I} \rightarrow I_{S}^{-}$.

Defect recombination $\left(J_{i}\right.$ and $\left.V_{J}\right)$ can occur via two channels (Fig.7): (1) a thermofluctuation jump through a constant recombination barrier $Q_{R}$ with probability $1 / \tau_{R 1} \approx$ $\omega_{D} \exp \left(-Q_{R} / k T\right), \omega_{D}$-frequency of Debye) and (2) recombination-stimulated removal of the barrier when capturing the electron and the hole at the local level, which is introduced by a close Frenkel pair into the forbidden band, with probability $1 / \tau_{R 2} \approx\left[1 / \sigma_{n} v_{n} \Delta_{n}+\right.$ 
$\left.1 / \sigma_{h} v_{h} \Delta p\right]^{-1}$ (where $v_{n}$ and $v_{h}$ are the thermal velocities of electrons and holes; $\sigma_{n}$ and $\sigma_{p}$ are sections for the capture of electrons and holes generated by the light onto the local level; $\Delta n$ and $\Delta p$ are the excessive concentration of non-equilibrium carriers). The complete probability for annealing of the Frenkel pairs is defined as $[14,15]$ :

$$
1 / \tau_{R}=1 / \tau_{R 1}+1 / \tau_{R 2}
$$

In general, the value of $1 / \tau_{R 2}$ will depend on the light pumping intensity $I: 1 / \tau_{R 2}=$ $=$ const $* I^{\alpha}$ (where $\alpha \approx 1$ or $1 / 2$ depending on the excess carrier recombination mechanism $[16])$. Then, the kinetics of the accumulation of close Frenkel pairs $\left[J_{i} V_{J}\right]$ is given by:

$$
\frac{d N_{d}}{d t}=N_{S}^{0} \sigma_{d} I-\frac{N_{d}}{\tau_{R}}
$$

which, at the initial condition of $t=0, N_{d}(0)=0$, has the solution:

$$
N_{d}=N_{S}^{0} \sigma_{d} I \tau_{R}\left[1-\exp \left(-t / \tau_{R}\right)\right] .
$$

Accordingly, for the steady state, we have a constant concentration of defects:

$$
N_{d}=N_{S}^{0} \sigma_{d} I \tau_{R}
$$

here, $N_{S}^{0}$ is the concentration of iodine nodes in the regular perovskite lattice. From (7), (4), and (2) follows the completely non-trivial dependence of the steady-state concentration of close Frenkel pairs on the pumping light intensity $N_{d}(\mathrm{I})$, since, as it follows from the above-stated, $\frac{d \sigma_{d}}{d I}<0$ and $\frac{d \tau_{R}}{d I}<0$ (Fig. 8).

Thus, by selecting the actual mode of light irradiation $\left(I<I_{\min }\right.$ and $\left.I>I_{\max }\right)$, one can reduce degradation to a minimum.

As for the comparison of the above theory with experimental data, then, due to the small number of experimental works (as noted, there is only one paper in which the degradation of perovskite solar cells has been detected [6]) one may as yet speak only of qualitative agreement between theory and experiment.

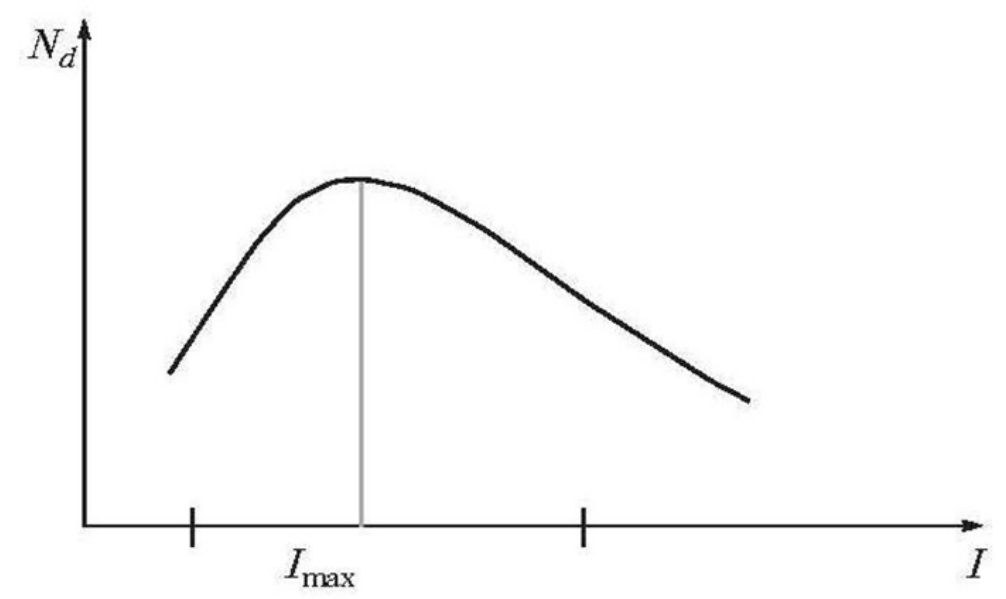

FIG. 8. The non-monotonic dependence of the concentration of Frenkel pairs in the I-sublattice on the intensity of radiation 


\section{Conclusions}

In this work, we constructed a model for radiation-induced deep shell ionization of the iodine ion followed by an Auger process, recharging, and rolling of the latter ion into the neigh-boring interstice. The effect of competition between the ion and electron relaxation in dynamically unstable structure of the local region of the lattice was taken into account to obtain a semi-quantitative analysis. In addition, the role of both thermal and recombination-stimulated annealing of the Frenkel pairs is explored. This shows that the degradation process of perovskite solar cells is based on the modified mechanism of Varley. The above results can be applied to wide spectrum problems (rather than ultraviolet irradiation), viz., the problem of radiation resistance in relation to ionizing radiation (Xrays, electrons, and $\gamma$-quanta). The observed non-monotonic dependence of the number of stationary defects should allow one to optimize conditions for reducing the radiation-based degradation of devices.

\section{References}

[1] Yin Wan-Jian, Yang J.-Hui, Kang J., et al. Halide perovskite materials for solar cell; a theoretical review. J. Mat. Chem. A. DOI: 10.10.39/c4ta05033a.

[2] Giorge G., Yamashita K. Organic-inorganic halide perovskites: an ambipolar class of materials with enhanced photovoltaic performances. J. Mat. Chem. A. DOI: 10.10.39/c4ta05046k.

[3] Frost J., Butler K.T., Brivio F., et al. Atomistic origin of high-performance in hybrid halide perovskite solar cell. arXiv: 1402.4980v3 (Cond mat. mtrl-sci) apr. 2014.

[4] Yin W.-J., Shi T., Yan Y. Unusual defect physics in $\mathrm{CH}_{3} \mathrm{NH}_{3} \mathrm{PbJ}_{3}$ perovskite solar cell absorber. Appl. Phys. Lett., 2014, 104, P. 063903.

[5] Oksengendler B.L., Ismailova O.B., Marasulov M.B., Urolov I.Z. On the degradation mechanism of functioning solar cells based on organic-inorganic perovskites. Appl. Solar Energy, 2014, 50(4), P. 255259.

[6] Burschka J., Pelle, N., Soon-Jin Moon, et al. Sequential deposition as a route to high-performance perovskite-sensitized solar cells. Nature, 2013, 499, P. 316-319.

[7] Itoh N., Stoneham A.M. Materials Modification by Electronic Excitation, Cambridge, Univ. Press, 2001.

[8] Oksengendler B.L., Turaeva, N.N. Radiatsionnaya fizika kondensirovannykh sred (Radiation Physics for Condensed Mediums), v. 1: Kontseptsii (Conceptions), Tashkent, Fan, 2006.

[9] Varley J. Discussion on Some Mechanisms of F- centre Formation in Alkali Hflides. I Phys. Chem. Sol., 1962, 23, P. 985-1005.

[10] Yunusov M.C., Abdurakhmanova S.N., Oksengendler B.L., et al., Fizicheskie svoistva obluchennogo kremniya (Physical Properties of Irradiated Silicon). Tashkent, Fan, 1987.

[11] Parilis E.E. Effekt Ozhe (Auger Effect). Tashkent, Fan, 1969.

[12] Yunusov M.S., Zaykovskaya M.A., Oksengendler B.L. et al. Subthreshold defect production in Si. Phys. Status Solidi A, 1976, 35, P. K145-149.

[13] Dexter D. Varley Mechanism of Defect Formation in Alkali Holides. Phys. Rev., 1960, 118(4), P. 934-935

[14] Yunusov M.S., Abdurakhmanova S.N., Oksengendler B.L. et al. Podporogovye radiatsionnye effekty $v$ poluprovodnikakh (Subthreshold Radiation Effects in Semiconductors). Tashkent, Fan, 1989.

[15] Yunusov M.S., Abdurakhmanova S.N., Oksengendler B.L. et al. The influence of minority carrier injection on subthreshold defects in Si. Phys. Status Solidi A, 1984, 81, P. K145-149.

[16] Davydov A.S. Teoriya tverdogo tela (Theory of solid state). Moscow, Nauka, 1976, 640 p.

[17] Toyozawa Y. Self-Trapping and Defect Reaction. Semicond and Insulators, 1985, 5, P. 175-200.

[18] Davison S., Levine Y. Surface (Tamm's) States. Mosc. Mir, 1973, p. 232.

[19] Schoonman. R. Organic - inorganic lead halide perovskite Solar cells materials. A possible stability problem. Chem. Phys.Lett., 2015, 619, P. 193-195.

[20] Itoh N. Nakayama T. Desorption from compound Semiconductors and Oxides induced by Laser irradiation. Semicond. and Insulators, 1983, 5, P. 383-400. 